\title{
CROCE TRIESTINA DI ALDA ÇULIANI E LE SUE “SORELLE” ZARATINE
}

\author{
Nikola Jakšić
}

\author{
N. Jakšić \\ Sveučilište u Zadru \\ Obala kralja Petra Krešimira IV. bb \\ 23000 Zadar, Croatia
}

\begin{abstract}
In Zadar area there are four silver processional crosses supporting crucified Christ's body that greatly resemble the famous Trieste example commissioned by Alda Çuliani in 1383. The discussion draws attention to the similarities and differences between the Zadar group and the cross in Trieste. Finally, it leads to the conclusion that the matrix for embossing Christ's body in medieval Zadar was used by two brothers, Stephen and Paul sons of Peter, goldsmiths, who moved from Cattaro around 1385.
\end{abstract}

Keywords: medieval goldsmith, silver crosses, Zadar, Trieste, Alda Zuliani, Stephan and Paul from Cattaro

I manufatti orafi trecenteschi conservati a Trieste non sono tanto numerosi. Due attirano l'interesse dello studioso, entrambi sono croci. Di una, custodita dalle suore benedettine presso il monastero di San Cipriano, scrissi un articolo nel 2010. In quell'occasione cercavo di dimostrare che la croce triestina faceva parte d'un gruppo di circa quindici prodotti d'arte applicata trecenteschi, distribuiti in un ampio territorio che va dal Veneto sino alla Dalmazia, eseguiti da orafi a noi sconosciuti, che usavano spesso un certo repertorio di matrici per fabbricare i particolari figurativi necessari a comporre una croce medievale, cioè i busti di dolenti, i simboli di evangelisti ed il corpo intero di Cristo crocifisso. ${ }^{1}$

In questa sede, dedicata all'amico Igor Fisković mi occupo della seconda croce trecentesca triestina (quella più bella e più famosa), nota sotto il nome della sua committente, Alda Çuliani. ${ }^{2}$ Nell'epigrafe votiva si legge l'anno 1383, cioè la data di esecuzione della croce ed il nome della nobildonna triestina che l'ha commissionata. (fig. 1) Tutti i due lati della croce riportano la medesima raffigurazione, il Cristo crocifisso. La parte posteriore reca la scena dipinta sul legno invece quella anteriore è rivestita di cinque lamine d'argento sbalzato che coprono l'intera anima di legno. Lo sfondo è punzonato a buccia d'arancia, ricoperto di motivi fitomorfici sbalzati, rami e foglie di quercia con delle ghiande. Nella placca centrale è raffigurato il Cristo crocifisso nimbato, ed accanto a lui i volti dei dolenti, la Madonna e San Giovanni Evangelista, ognuno sulla sua placca separata. Sulla sommità del braccio verticale è raffigurato il busto di San Michele Arcangelo, mentre la figura della donatrice Alda Çuliani è ritratta di profilo ai piedi del Cristo, velata, inginocchiata e orante, con le mani giunte nel gesto della preghiera. Sotto le sue ginocchia, in fondo del braccio verticale, sullo sfondo niellato si trova l'iscrizione votiva in due righe:

\section{DO/NNA ALDA ÇULIAN}

\footnotetext{
${ }^{1}$ N. JAKŠSIĆ, Rapporti veneto-dalmati nell'oreficeria trecentesca in Letteratura, arte, cultura tra le due sponde dell'Adriatico, (a cura di N. Balić Nižić, N. Jakšić, Ž. Nižić), Zadar 2010, 299 - 326.

${ }^{2}$ Questa relazione è stata letta nella giornata di studio Letteratura, arte, cultura italiana tra le due sponde dell'Adriatico tenurasi a Padova in autunno 2009.
} 


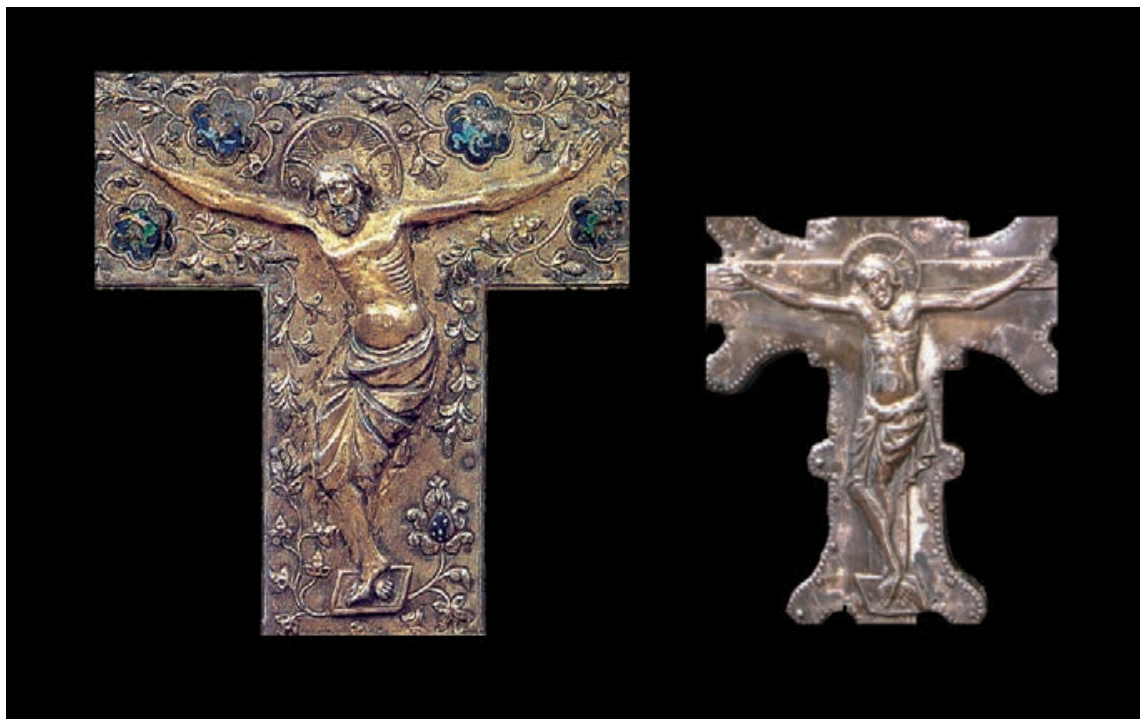

Fig. 1. Particolari con i corpi di Cristo nelle due croci triestine (di Alda Çuliani - sinistra; San Cipriano destra), rapporto di formati.

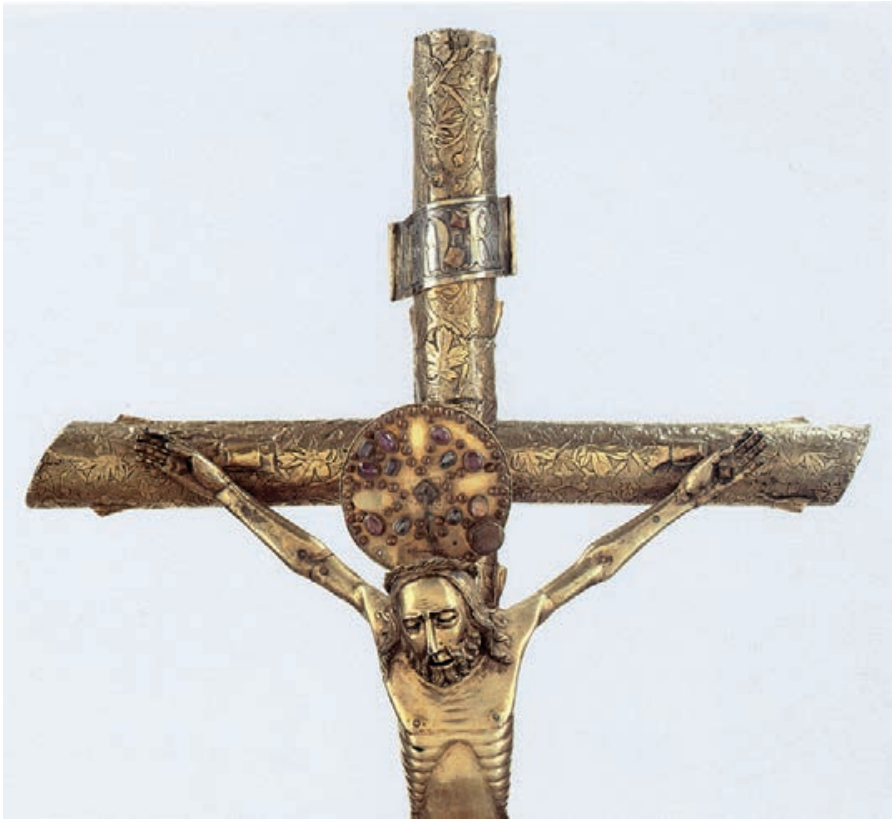

Fig. 2. Croce dei Principi, particolare, Gorizia, tesoro della cattedrale metropolitana.

drale di Corizia (appartenente in origine ai patriarchi aquileiesi) e alla croce di Alda Culiani. 5 Ciad titolo della relazione si desume che l'interesse della studiosa è orientato verso l'analisi dell'iconogra-

\footnotetext{
${ }^{3}$ L. CRUSVAR, Il drago alato nell'iconografia del basso Medioevo e alcune sue rivisitazioni fra Trieste, la Dalmazia e le terre veneziane, Società istriana di archeologia e storia patria 52, Trieste 2004, pp. 159 . 213.

${ }^{4}$ L. CRUSVAR, Gli argenti ecclesiali a Trieste: settore ancora inesporato in tesori delle communità religiose di Trieste (catalogo della mostra), Udine 1978, pp. 15 - 17; Medioevo a Trieste (catalogo della mostra) Silvana Editoriale, Milano 2008, p. 183 (scheda: A. Krekic).

${ }^{5}$ L. CRUSVAR, La croce della vita nell'iconografia del XIV secolo. Due esempi dell'arte orafa tra Venezia, Aquileia e Trieste, in In hoc signo - Il tesoro delle croci (a cura di P. Goi), Skira 2006, pp. 94 - 107.
} 
fia specifica dei preziosi manufatti. Tutti e due portano la rappresentazione della croce con le travi dalle quali spuntano i fiori. Il patibulum, lo strumento di martirio, il legno di morte al quale è appeso Cristo, in contatto con il corpo umano/divino diventa vivo. Nell'esemplare goriziano due tronchi massicci d'albero, senza dubbio di quercia, nodosi, formano i bracci della croce ricoperti con un decoro a tralci di vite (fig. 2). ${ }^{6} \mathrm{La}$ Croce triestina invece è tutta ricoperta di rami e di foglie di quercia con delle ghiande. Lo strumento del martirio qui non è raffigurato, come invece sul verso, ed è presente soltanto nella sagomatura del manufatto integro. Tuttavia i piedi di Cristo appoggiano su suppedaneo.

Prendendo in esame il problema iconografico, la Crusvar ci rimanda a noti esemplari d'arte figurativa recanti delle rappresentazioni di croci - strumenti di martirio, interpretati come l'albero vivo e fruttifero.

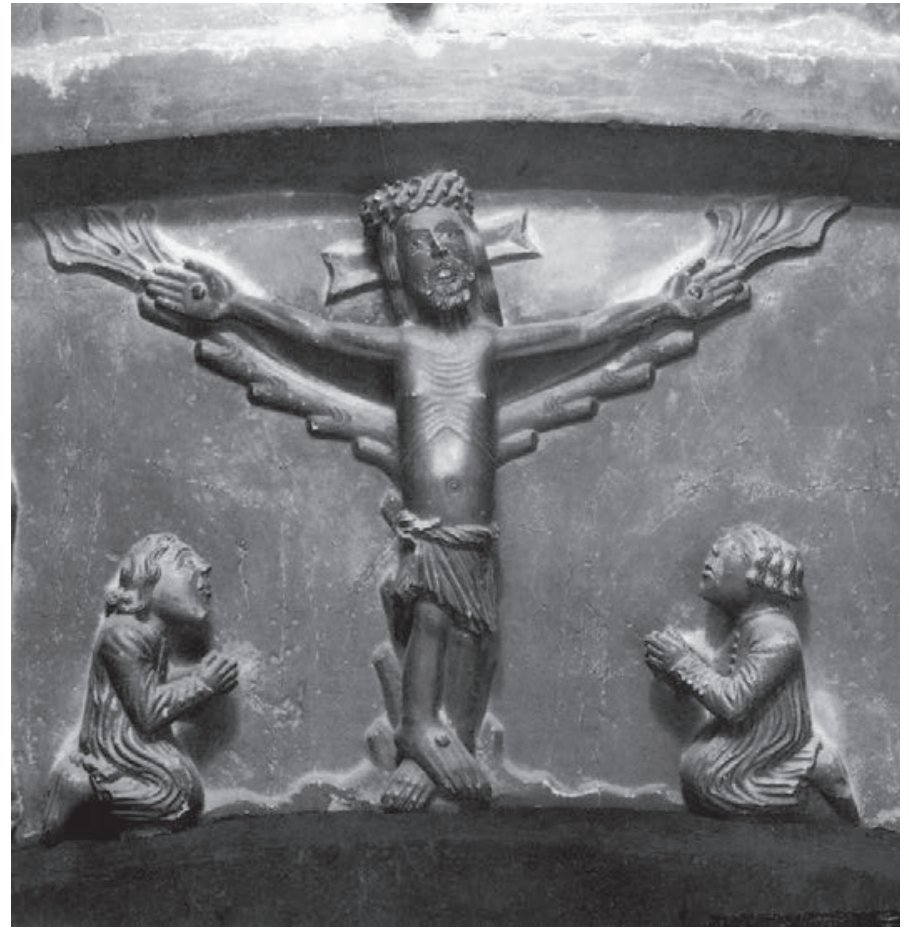

Fig. 3. Crocifissione sul serraglio dell'arco a Traù

Dal famoso rilievo di Benedetto Anelami

a Parma ci porta fino alle rappresentazioni nella pittura trecentesca, dagli affreschi a Sesto al Reghena, a Udine, a santa Croce a Firenze ${ }^{7}$. Nella spiegazione del fenomeno della croce interpretata nelle arti visive nell'epoca, del Trecento, come un albero vivo e fruttifero, la studiosa ci avvia al libro $L i$ gnum vitae di Bonaventura da Bagnoregio, ministro generale dell'ordine francescano dal 1257 al 1274 uno di quei teorizzatori della teologia della storia vista come progressiva avanzata della salvezza, e al libro Arbor vite crucifige Christi di Umbertino di Casale (+1310) uno di teoretici più estremi dell'ala spirituale del francescanesimo. Ci porta alla conclusione dunque, che tale interpretazione della croce si è ispirata alla teologia francescana. ${ }^{8}$

Anche sulla sponda adriatica orientale s'incontrano esemplari di questa tipologia di croce rappresentata come lignum vitae, sebbene raramente. Per questo motivo vale la pena richiamare l'attenzione al rilievo rappresentante la Crocifissione con i committenti inginocchiati, scolpito sul serraglio dell'arco esterno della cattedrale di Traù (fig. 3). A cavallo tra Duecento e Trecento, un maestro mediocre e ignoto, scolpì la Crocifissione, compresi i committenti inginocchiati che fiancheggiano il Cristo crocifisso sul tronco nodoso e fogliato, sagomato con uno schema a "Y". ${ }^{9}$ Ma la rappresentazione simbilica più antica di lignum vitae con gemme orna un sarcofago carolingio custodito nella chiesa parrocchiale a Valle, in Istria (fig. 4). Scrivendo sul sarcofago di Valle, B. Fučić ${ }^{10}$ non ha dimenticato

\footnotetext{
${ }^{6}$ Bibliografia completa sulla "croce dei Principi“" vedi in In hoc signo - Il tesoro delle croci (a cura di P. Goi), Skira 2006, p. 364 (scheda: L. Crusvar)

${ }^{7}$ Ivi, pp. 99 - 101

${ }^{8}$ Ivi, pp. $99-101$.

${ }^{9}$ Su portale di Traù vedi C. FISKOVIĆ, Radovan, Zagreb 1989, ed interventi di vari autori nella racolta: Majstor Radovan i njegovo doba (a cura di I. Babić), Trogir 1994.

${ }^{10}$ B. FUČIĆ, Sarkofag iz Bala, Peristil 29, Zagreb, 1986, pp. 23 - 26; L'esemplare da Valle non è isolato in vasto corpus della scultura carolingia. Ricordo ad un pluteo dalla cattedrale di S. Anastasia a Zara che dimostra l'identica iconografia, vedi N. JAKŠIC - E. HILJE, Kiparstvo I, - Umjetnička baština Zadarske nadbiskupije, Zadar 2006, pp. 97 - 100.
} 


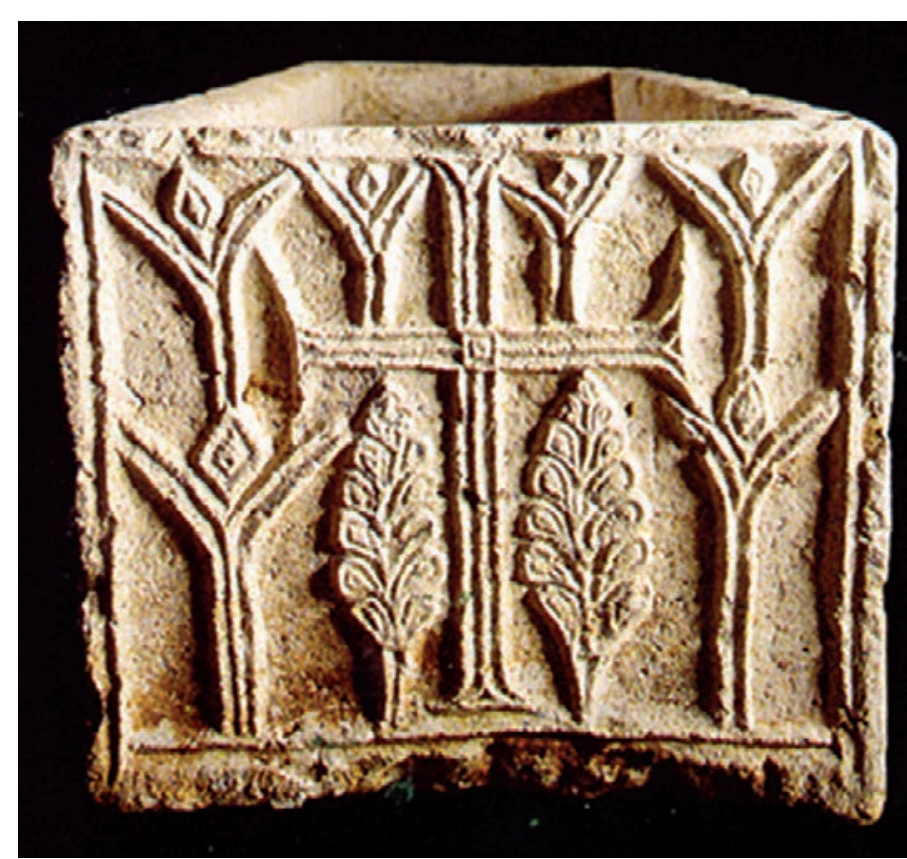

Fig. 4. Lato del sarcofago carolingio a Valle

di sottolineare il testo del ben noto inno di Venantio Fortunato (530 - 607) Lustra sex qui jam peregit che faceva parte di quasi ogni innario medievale e che viene usata specialmente nelle celebrazioni della liturgia del Venerdì Santo:

\section{CRUX FIDELIS, INTER OMENES ARBOR UNA NOBILIS: SILVA TALEM NULLA PROFERT FRONDE, FLORE, GERMINE: DULCE FERRUM, DULCE LIGNUM, DULCE PONDUS SUSTINENT. ${ }^{11}$}

Forse proprio in quel cantico composto da un poeta a noi geograficamente tanto vicino, perché Fortunato nacque vicino a Treviso, si riconosce la fonte letteraria che potrebe favorire le visualizzazioni del tipo della croce in questione. Senza dubbio la teologia francescana aiutò la sua diffusione nelle arti visive, ma ci sono numerosi esemplari di lignum vitae nell'arte eseguiti di fuori del contesto francescano. Bastarebbe ricordare il famoso mosaico in San Clemente a Roma con la croce che emerge da cespuglio e domina l'abside tutta ricoprta di girali di foglie d'acanto. ${ }^{12}$ Questa "abside-staurotheca", nota Valentino Pace "non inverosimilmente pote riprendere proprio il sintagma croce-vegetazione dal mosaico della chiesa paleocristiana." 13 L'esemplari argentei partono dal 6 secolo come lo dimostra la famosa staurotheca cosidetta "Crux Vaticana" nel Museo Storico Artistico del Tesoro di San Pietro, commissionata da Giustino II (565 - 578). All'incrocio dei bracci è colocata una capssela che contiene la reliquia della Vera Croce, e le braccia sono caraterizzate da quattro lamine d'argento sbalzate, decorate da fogliami di palmete e cornucopia. "Cosi la Santa Croce del Cristo si transforma misticamente in Arbor vitae," sottolinea Sante Guido. ${ }^{14}$ In particolare penso che sia utile avvertire ad una croce astile attribuita all'aurifaber Perrone Malamorte, sbalzata in maniera bizantina nei primi decenni del Duecento, cioè in tempo di Federico II, conservata nel tesoro della cattedrale di Messina, le cui braccia sono quasi interamente coperte di palmette ${ }^{15}$. (fig. 5)

Tuttavia in seguito vorrei ricordare soltanto alcuni esemplari conservati nella zona altoadriatica provenienti da: Valle ${ }^{16}$, Fianona ${ }^{17}$, Buccaro ${ }^{18}$, Pago $^{19}$ e Soline ${ }^{20}$ nella sponda orientale del Adriatico; da

\footnotetext{
${ }^{11}$ http://www.preces-latinae.org/thesaurus/Hymni/PangeF.html

${ }^{12}$ S. RICCIONI, Il mosaico absidale di S. Slemente a Roma. Exemplum della chiesa Riformata, CISAM, Spoleto 2006.

${ }^{13}$ V. PACE, La Crux Vaticana e la Roma bizantina in La Crux Vaticana o Croce di Giustini II, Edizioni Capitolo Vaticano 2009, p. 6.

${ }^{14}$ S. GUIDO, Nuovi dati e osservazioni tecniche emerse dalle operazioni di restauro in La Crux Vaticana o Croce di Giustini II, Edizioni Capitolo Vaticano 2009, p. 12.

${ }^{15}$ M. ACCASCINA, Oreficeria di Sicilia dal XII al XIX secolo, Palermo 1974, p. 96.

${ }^{16}$ A. SANTANGELO, Inventario degli oggetti d'arte d'Italia V, Provincia di Pola, Roma 1935, p. 119; P. PAZZI, Itinerari attraverso l'oreficeria veneta in Istria e Dalmazia, Treviso 1994, p. 26.

${ }^{17}$ A. SANTANGELO, Inventario degli oggetti..., cit., p. 9; Argenteria liturgica dell'Albonese, (ed. Tullio Vorano), Labin 1993, no. 26.

${ }^{18}$ M. KOVAČEVIĆ, Nekoliko priloga gotičkom zlatarstvu vinodolskog kraja Radovi Instituta za povijest umjetnosti 29, Zagreb 2005, pp. 17 - 23; N. JAKŠIĆ, Rapporti veneto-dalmati nell'oreficeria trecentesca cit., p. 314.

${ }^{19}$ N. JAKŠIĆ - R. TOMIĆ, Zlatarstvo - Umjetnička baština Zadarske nadbiskupije, Zadar 2004, pp. 193 - 195.

${ }^{20}$ Ivi, pp. $146-147$.
} 
Villorba (TV), ${ }^{21}$ Camposampietro (Pd), ${ }^{22} \mathrm{Ze}$ vio $(\mathrm{Vr})^{23}$, Marcon $(\mathrm{Ve})^{24}$, Monselice $(\mathrm{Pd})^{25}$ in Veneto, da San Giovanni in Bragora e da Santi Geremia e Lucia a Venezia ${ }^{26}$, compresi naturalmente quelli di Alda Giuliani a Trieste e "dei Principi" da Aquileia. Un gruppo distinto è rappresentato dalle croci veneziane il cui corpo in cristalo di rocca è delimitato da una cornice in argento dorato da cui si staccano girali a riccioli portanti montature a bocciolo con ghiande. Quella della Scuola Grande di San Giovanni Evangelista $^{27}$ fu un prototipo imitato più volte durante il Quattrocento nella città lagunare. ${ }^{28}$ L'esemplare simile proveniente dalla chiesa di Sant'Agata dei Teatini a Bergamo è stato avvicinato alla bottega dei Da Sesto ${ }^{29}$. L'unica croce di questo tipo a me nota fuori

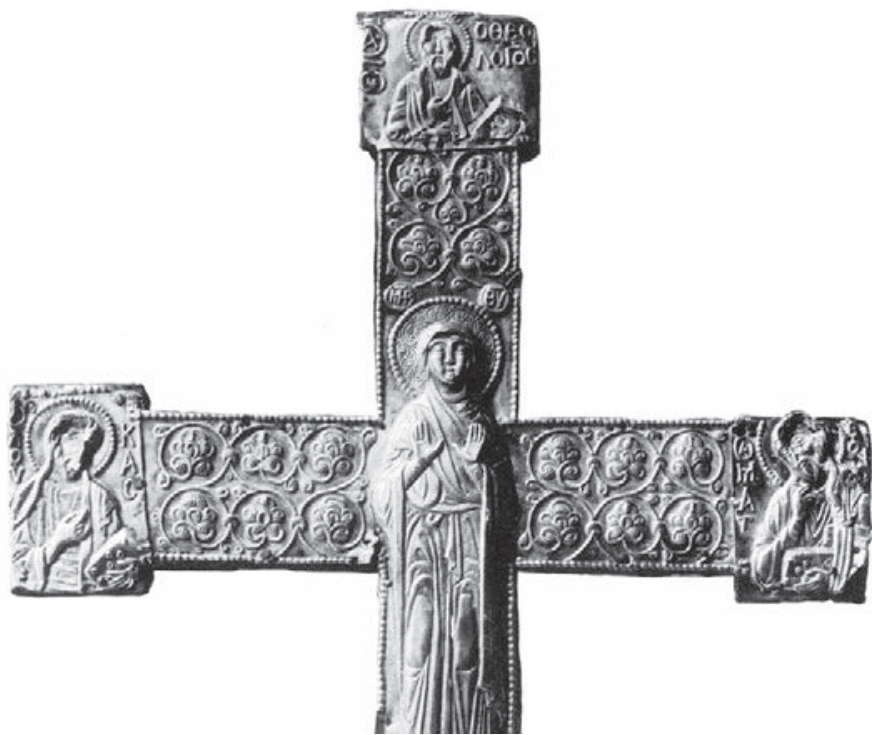

Fig. 5. Perrone Malamorte, la croce a Messina, particolare dallo stato di Venezia si trova a Ragusa, commissionata nel 1440 dai francescani locali da un orafo girovago, Johannes de Basileia ${ }^{30}$.

Ma per il momento mettiamo da parte il problema della croce gemmata e fiorente. Vorrei parlare principalmente della tipologia del corpo di Cristo fissato e appeso allo strumento del martirio, il che costituisce è il particolare più interessante della croce di Alda Çuliani. Nella sua relazione L. Crusvar sottolinea: il Christus patiens che campeggia al centro della montatura argentea, rimanda "ad un modello e a uno stampo largamente utilizzato in tutta l'area di influsso veneziano, verificabili in tre croci dalmate pubblicate da prof. Petricioli," conservate in alcune località attorno a Zara (Nona, Božava, Ugljan). Dice in seguito che questo modello "si ripropone pure nel Cristo dolente" su un altra croce a Trieste, quella nel monastero di San Cipriano ${ }^{31}$.

Secondo me, il corpo di Cristo dalla croce custodita nel monastero di San Cipriano a Trieste non appartiene a gruppo summenzionato. Prima di tutto è più piccola della croce di Alda Çuliani (fig. 1). Tra l'altro la croce triestina da San Cipriano fa parte di un gruppo diverso, omogeneo ed abbastanza numeroso, già discusso nella letteratura settoriale ${ }^{32}$.

\footnotetext{
${ }^{21}$ Oreficeria sacra in Veneto - secoli VI - XV, (a cura di A. M. Spiazzi), Biblos Edizioni Cittadela 2004, pp. 158 - 159 (scheda: G. Delfini Filippi)

${ }^{22}$ Ivi, p. 159 - 161 (scheda: G. Ericani)

${ }^{23}$ Ivi, pp. 164 - 165 (scheda: A. Malavolta)

${ }^{24}$ Ivi, p. 166 - 167 (scheda: D. Samadelli)

${ }^{25}$ Ivi, pp. 183 - 184. (scheda: G. Ericani)

${ }^{26}$ G. M. PILO, Oggetti veneziani di oreficeria sacra dal XIV al XVI secolo, in Ori e tesori d'Europa (a cura di G. Bergamini e P. Goi), Udine 1992, 65; G. CAPUTO, La Croce, il Crocifisso e il Mistero della Risurrezione, in In hoc signo - Il tesoro delle croci (a cura di P. Goi), Skira 2006, pp. 235 - 241.

${ }^{27}$ HAHNLOSER, R. H. - BRUGGER-KOCH, S., Corpus der Hartsteinschliffe des 12.-15. Jarhrunderts, Berlin 1985, p. 126, scheda 140.

${ }^{28}$ Ivi, p. 120, scheda 122 (scuola di San Giorgio degli Schiavoni); p. 120, scheda 123 (Santa Maria della Salute); p. 121 scheda 124 (Scuola di San Teodoro)

${ }^{29}$ Ivi, p. 120, scheda 121.

${ }^{30}$ N. JAKŠIĆ, Raspeće-relikvijar kod male braće u Dubrovniku, Ars Adriatica 2, Zadar 2012, 109 - 118.

${ }^{31}$ L. CRUSVAR, La croce della vita nell'iconografia del XIV secolo, cit. p. 101. La studiosa sfortunamente non fa alcun riferimento alla letteratura croata dove le sopradete croci zaratine furono pubblicate più di 30 anni fa.

${ }^{32}$ N. JAKŠIĆ, Rapporti veneto-dalmati nell'oreficeria trecentesca..., cit., pp. 299 - 326.
} 

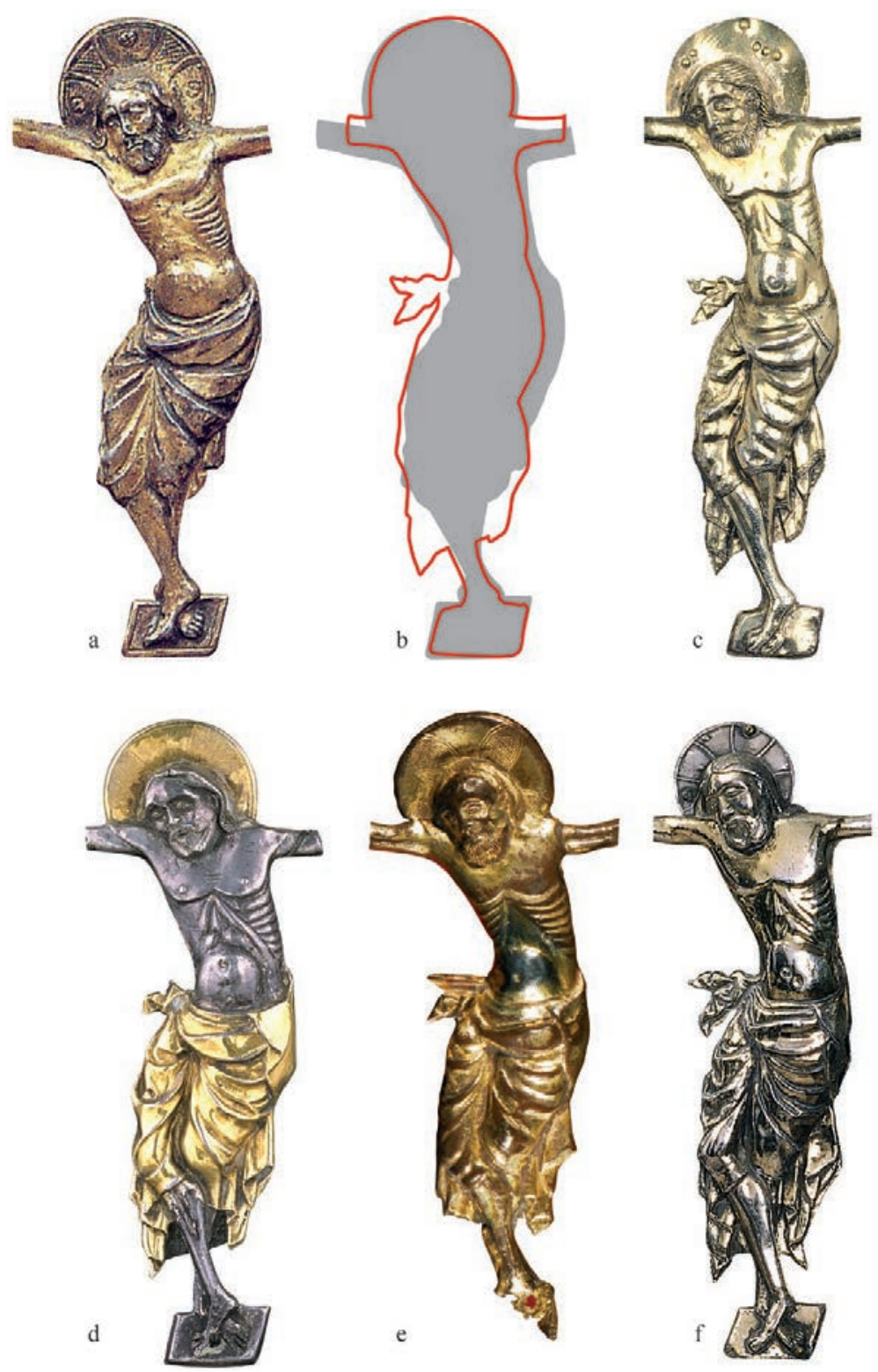

Fig. 6. Corpo di Cristo dalla croce di Alda Culiani in rapporto agli esemplari zaratini (a. Alda Zuliani, b. disegni sovraposti - rosso esemplari zaratini, c. Božava, d. Kale, e. Ugljan, f. Nona)

Tre croci dall'area zaratina che L. Crusvar collega a quella di Alda Çuliani furono individuate da prof. Petricioli nel 1963, ma in tale momento lui non ha proposto alcun paragone alla nota croce triestina. ${ }^{33}$ In una relazione del 1999, (mai pubblicata) Petricioli aggiunse al gruppo anche l'esemplare dalla chiesa parrocchiale di San Lorenzo a Kale un villaggio insulare di fronte a Zara. ${ }^{34}$ In tale occasione Petricioli ha sottolineato che il corpo di Cristo dalla croce di Alda Çuliani servì come modello per tutto il gruppo dei corpi dell'area zaratina, proposta evidentemente accettata da L. Crusvar. Anche io avevo accettato questa opinione con cautela, sottolineando tuttavia che si tratta di una matrice simile, ma non del tutto identica ${ }^{35}$. Per risolvere il dilemma sul carattere della matrice (identica o sol-

\footnotetext{
${ }^{33}$ I. PETRICIOLI, Prilozi izučavanju srednjovjekovnog zlatarstva u Zadru, Radovi Filozofskog fakulteta u Zadru 2, Zadar, 1963, 132 - 146.

${ }^{34}$ Per dire la verità Petricioli in tale mommento aggiunse al gruppo due esemplari (quasi identici) da Kali perché non se ne accorse che soltanto una fu la croce medievale, quella sbalzata, invece l'altra e un esemplare fuso (che è coppiato dall'originale) commissionato dalla fraternità di Santa Trinità nella seconda mettà del ottocento.
}

${ }^{35}$ N. JAKŠIĆ - R. TOMIĆ, Zlatarstvo - Umjetnička baština Zadarske nadbiskupije, cit. p. 154. 

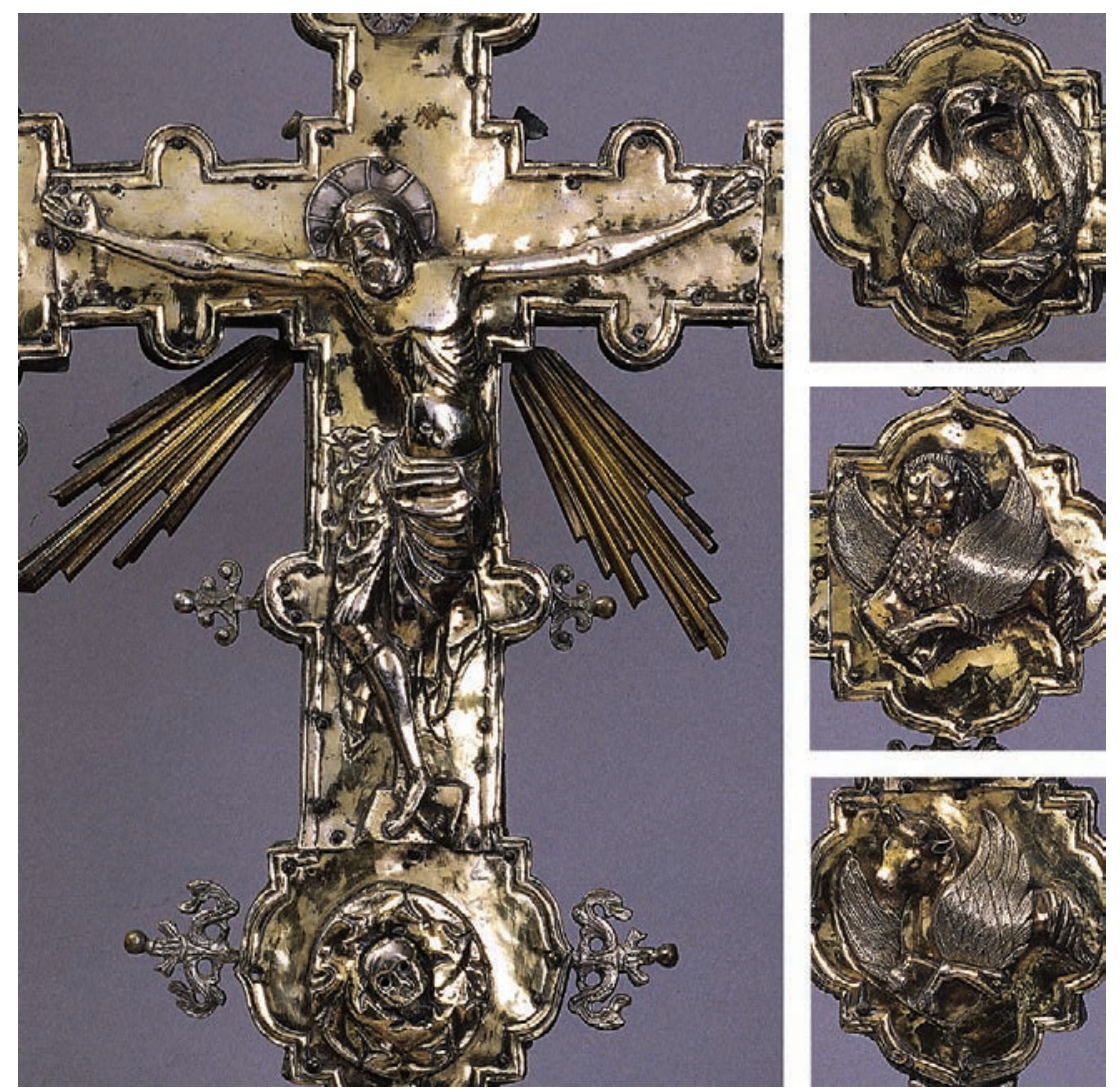

Fig. 7. Croce a Nona, particolari di qualità abbastanza alta, attribuiti all'orafo Stefano di Pietro da Cattaro, prima del 1405

tanto simile) che è servita per sbalzare i corpi di Cristo, ho preparato un disegno con le sagomature dei corpi di Cristo nell'esempalre zaratino e in quello triestino. (fig. 6) I disegni sovrapposti dimostrano un significativo grado si somiglianza ma anche delle differenze notevoli che ci confermano che le matrici usati a Zara e a Trieste non erano del tutto identiche. Nel esemplare triestino il fianco sinistro del corpo di Cristo è spostato di più indietro. Per quanto riguarda il perizoma, quello zaratino ha le pieghe più lunghe che raggiungono quasi le caviglie del corpo crocifisso e sul fianco destro un nodo con un ricco fiocco; tutto ciò non esiste nell'esemplare triestino.

Nonostante tutte le differenze menzionate è evidente che il perizoma zaratino deriva da quello triestino. Si tratta in particolare del trattamento molto specifico del panneggio incastrato tra le cosce di Cristo, creando le pieghe radialmente distribuite da quel punto. Si deve pertanto concludere che la matrice usata dai maestri zaratini dipendeva direttamente dalla croce triestina di Alda Çuliani, ma anzi che nella preparazione della matrice zaratina l'attenzione particolare è stata dedicata al perizoma i cui panneggi diventano più svariati, più ricchi, più agili, cioè più naturalistici. Ci sorprese anche il fatto che i corpi di Cristo a Zara ed a Trieste hanno delle misure uguali, sono alti $20 \mathrm{~cm}$.

Di quattro esemplari zaratini in questione, tre sono conservati nei villaggi dalle isole zaratine (Ugljan, Božava, Kale) mentre il quarto proviene da Nona ${ }^{36}$, cittadina vicino a Zara. L'ultimo si distingue dagli altri tre perché porta il nome dell'orafo che lo sbalzò. L'epigrafe +STE/FANUS/FECI(T) è collocata sulla mensola poligonale che sostiene la figura di San Martino vescovo fissato al centro della parte posteriore della croce. Nonostante questa importante informazione lo stile dell'orafo Stefano per il momento rimane nascosto perché l'esecuzione di questa croce a Nona dimostra due diversi livelli di qualità; il corpo di Cristo e simboli di tre evangelisti (l'aquila, il leone ed il bove) sono voluminosi, sbalzati usando matrici di qualità abbastanza alta (fig. 7). Al contrario i busti dei dolenti, cioè la Ver-

${ }^{36}$ Dal 9 secolo fino al 1828 sede della omonima diocesi. 

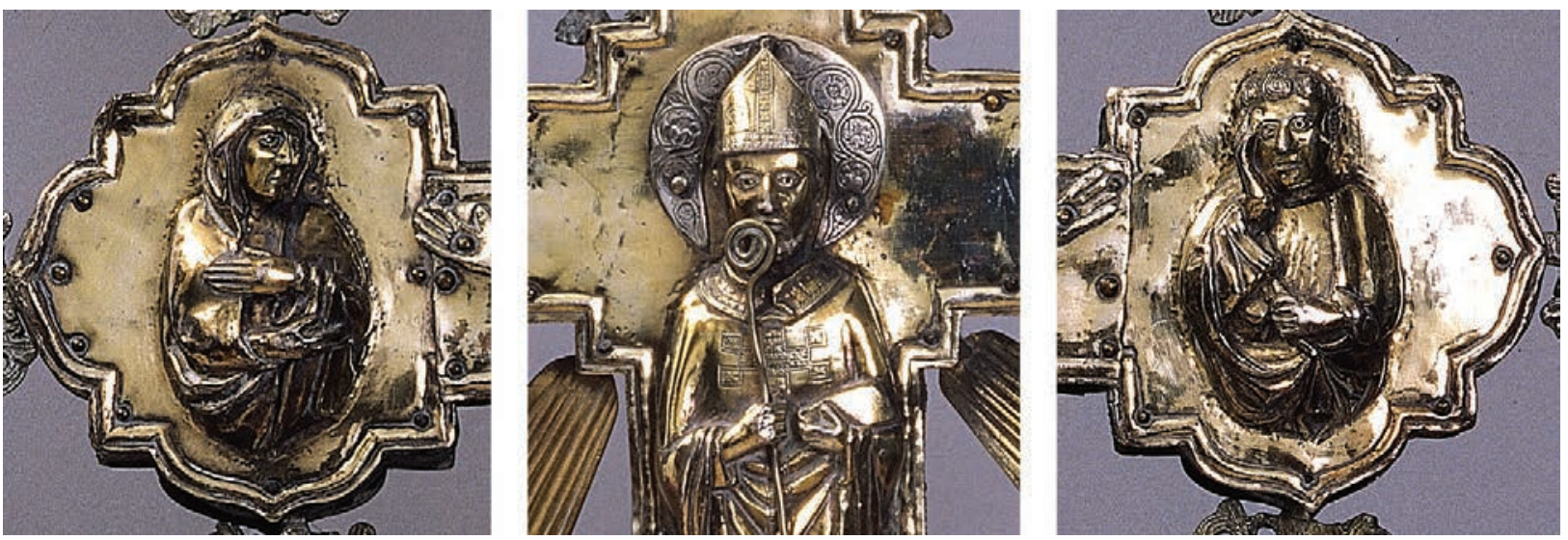

Fig. 8. Croce a Nona, particolari di qualità mediocre, attribuiti all'orafo Stefano di Pietro da Cattaro, prima del 1405

gine e San Giovanni Evangelista, e l'immagine di San Martino vesovo e dell'evangelista Matteo sono flaccidi, ed esprimono debolezza nel volume e nei movimenti goffi (fig. 8). Diventa evidente che la qualità artistica dell'opera dipendeva principalmente dalle qualità delle matrici che l'orafo possedeva, cioè che aveva a portata di mano. Nel caso in cui gli mancavano delle matrici, l'orafo fu costretto a crearle personalmente. Perciò si apre il problema legato alla vera paternità artistica del orafo Stefano. Quali particolari lui ha creato personalmente? Sembra quelli peggiori. Infine, la sua firma è collocata sotto la figura mediocre di San Martino.

Il secondo problema è legato alla datazione delle quattro croci zaratine. In ogni caso esse sono state sbalzate dopo 1383, l'anno della esecuzione della croce di Alda Çuliani. Verosimilmente a cavallo del secolo, verso l'anno 1400. Sembra che tale ipotesi trovi la sua prova in un documento scritto a Zara nel primo decennio del quattrocento. Il 27 luglio del 1407, un certo Cressiava Gliubanich (un nome croato), gastaldo della chiesa di San Nicolo a Božava (una delle località menzionate dove è conservata la croce), ha commissionato una croce argentea dall'orafo zaratino Paolo di Pietro. La croce doveva pesare 36 once d'argento e doveva essere dotata di un supporto con il nodo (pomo) di rame. La croce completa doveva essere dorata e tutto questo per un prezzo di 200 libbre di denari piccoli. ${ }^{37}$

È molto probabile che si tratti della croce dalla chiesa di San Nicolò a Božava conservatasi fino ai giorni nostri e oggi esposta nel museo diocesano a Zara (fig. 9) (Stalna izložba crkvene umjetnosti). Nella parte posteriore della croce campeggia la figura di San Nicolò il protettore celeste di questa piccola comunità cristiana fin dal periodo medievale. ${ }^{38}$

Cosi diventa evidente che la matrice per l'esecuzione del corpo di Cristo di ambito zaratino viene usata da due orafi diversi: da Stefano, per l'esecuzione della croce a Nona e da Paolo di Pietro per l'esecuzione della croce a Božava.

Paolo di Pietro fu un orafo trasferitosi a Zara da Cattaro, il cui nome nei documenti zaratini apparve per la prima volta nel $1385 .{ }^{39}$ Invece Stefano il cui nome viene sbalzato sulla croce di Nona può

\footnotetext{
${ }^{37}$ 1407, 27 luglio, Cressiava Gliubanich de Bosava tamquam gastaldo ut asseruit ecclesie Sancti nicolai de Bosava ex una parte et Paulus aurifex quondam Petri de jadra ex altera parte talia pacta cum infrascriptis modi set condicionibus ad invicem contradixerunt. Videlicet quod dictus Paulus promisit eidem Cressiave dicto nomine stipulanti facere et perficere infra sex menses proxime futuros unam crocem de argento lige veneziane ponderis unciarum triginta sex dicti argenti sine pomo et etiam facere popmum siffucientem di ramo et deaurare crucem et pomum suficienter et bene et hec omnia omnibus expensis dicti Pauli. Et hoc pro pretio librarum ducentarum.... (Archivio dello Stato a Zara, Archivio notarile, Petrus de Sarçana Busta V, fasc. 105.)

${ }^{38}$ N. JAKŠIĆ - R. TOMIĆ, Zlatarstvo - Umjetnička baština Zadarske nadbiskupije, cit., pp. 152 - 153.

${ }^{39}$ Un orefice Paolo condam Petri è menzionato in un documento notarile zaratino del 1377 (Archivio dello Stato a Zara, Archivio notarile, Petrus de Sarçana BustaII, fasc. 8, f. 5) ma considerando il fatto che non vi è alcuna indicazione di Cattaro come il suo luogo di origine, puo essere un altro orefice a noi scognosciuto.
} 


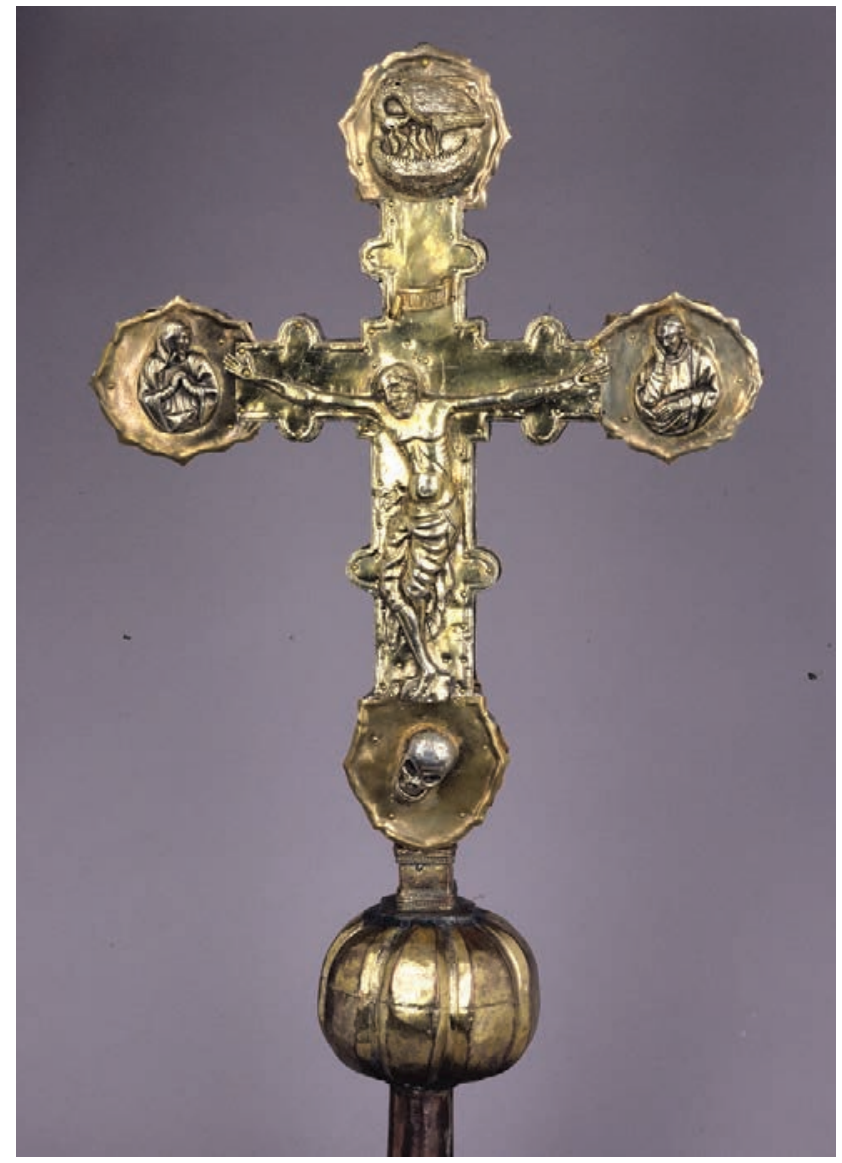

Fig. 9. Croce a Božava, attribuita all'orafo Paolo di Pietro da Cattaro, 1407

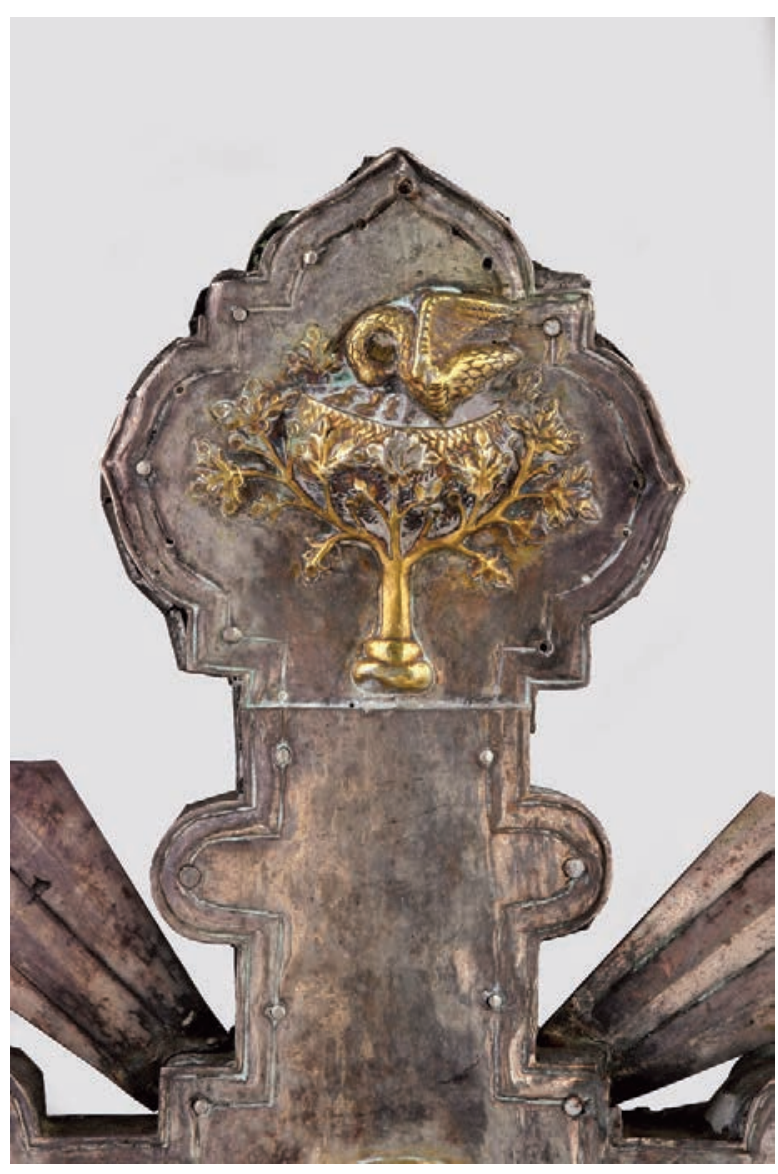

Fig. 10. Pellicano che ferisce il proprio petto, croce a Kale, particolare

essere qualsiasi Stefano orafo attivo a Zara alla fine del trecento, cioè: Stephanus aurifex condam Petri de Lapac, Stephanus aurifex condam Cernote uocato François, Stephanus aurifex condam Petri da Cattaro. ${ }^{40}$

Tuttavia i documenti storici indicano che l'orafo Stefano da Cattaro fosse il fratello del orafo Paolo da Cattaro. Tutti e due avevano il padre che nei documenti viene notato come fu Pietro. Nonostante che fin ora nessun documento afferma che questi due davvero fossero fratelli, il modo in cui sono nominati nei documenti Stefanus aurifex condam Petri da Cattaro e Paolo aurifex condam Petri da Cattaro lascia pochi dubbi. ${ }^{41}$

Paolo di Pietro che ha sbalzato la croce nel 1407 visse a Zara per un periodo abbastanza lungo, fino alla sua morte negli anni trenta del quattrocento. Stefano di Pietro invece non visse cosi lungo. Nel 1405 un documento menziona la sua vedova Margarita ${ }^{42}$ mentre personalmente Stefano fu menzionato vivo l'ultima volta nel anno $1401 .^{43}$

Se la mia ipotesi sulla relazione famigliare dei due orafi provenienti da Cattaro fosse valida, diventerebbe evidente che la croce a Nona, firmata da Stefano, fu sbalzata prima di quella di Božava commissionata a Paolo nel 1407.

\footnotetext{
${ }^{40}$ L'elenco dei orafi zaratini nel epoca medievale vedi in C. FISKOVIĆ, Zadarski sredovječni majstori, Split 1951, 107 - 131; N. KLAIĆ - I. PETRICIOLI, Zadar u srednjem vijeku, Zadar 1976, 531 - 543.

${ }^{41}$ N. JAKŠIĆ, Sakralno zlatarstvo Kotora u razvijenom srednjem vijeku, Zagovori svetom Tripunu (a cura di. R. Tomić), Zagreb 2009, 120.

${ }^{42}$ Archivio dello Stato a Zara, Archivio notarile, Iohannes de Trottis, B un, F I/1, f. 28.

${ }^{43}$ Archivio dello Stato a Zara, Archivio notarile, Articucius de Rivignano, B V, F II, no. 39 f. 1; N. JAKŠIĆ - R. TOMIĆ, Zlatarstvo - Umjetnička baština Zadarske nadbiskupije, cit. p. 29 nota 64.
} 
Il gruppo delle croci zaratine caratterizzate dai corpi di Cristo confrontabili a quello dalla croce di Alda Çuliani presentano, rispetto agli esemplari trecenteschi, una nuova soluzione iconografica. Non seguono più lo schema iconografico bizantineggiante con l'arcangelo che campeggia sulla parte superiore della croce e con Adamo in basso che esce dal sarcofago, sotto i piedi di Cristo. Il busto dell'arcangelo è sostituito da un pellicano che ferisce il proprio petto per nutrire i suoi pulcini, scena che simboleggia il sacrificio di Cristo. La versione di questa scena nella croce a Kale con il nido nidificato sui rami dell'albero è abbastanza unica nel suo genere (fig. 10). La scena con Adamo nel sarcofago invece, seguendo l'interpretazione leggendaria medievale secondo cui la Vera Croce fu eretta sulla tomba di Adamo, viene sostituita di un cranio entro la roccia. Questo mutamento di iconografia nella Zara medievale è presente per la prima volta proprio nel gruppo dei croci qui discusso, vuol dire a cavallo dei secoli XIV e XV. ${ }^{44}$ In quel momento ne Zara ne Trieste facevano parte della Serenissima perciò è inadequata l'osservazione della Crusvar che scrisse di "un modello e stampo largamente utilizato in tutta l'area di influsso veneziano."

\section{TRŠĆANSKI KRIŽ ALDE ZULIANI I ZADARSKI MU „SRODNICI“}

U trezoru katedrale Sv. Justa u Trstu čuva se vrijedan primjerak srednjovjekovnog zlatarstva. Riječ je o križu koji je posvetnim natpisom u nielo tehnici datiran u 1383. godinu. Nastao prema naruđ̋i lokalne plemkinje Alde Zuliani, iskucan je u srebru i pozlaćen. Stoga je podno raspetog Krista prikazan klećeći lik donatorice. Cijela je površina križa prekrivena biljnom viticom s pupoljcima i obogaćema emajlima. U svojoj raspravo o ovome i nekim drugim križevima, primjerice o Croce dei Principi, L. Crusvar započinje raspravu o ikonografiji križa lignum vitae, nalazeći da je toj tipologiji znatno pogodovala nova franjevačka teologija.

Autor međutim, upozorava da su križevi s temom lignum vitae zastupljeni u ikonografiji davno prije franjevačkog pokreta pa u tom smislu upozorava na neke poznate nadasve poznate primjerke kao što je primjerice križ Justina II (565 - 578) u Vatikanu, na jedan bizantinizirajući primjerak u Mesini ili pak na čuvenu apsidalnu mozaičku kompoziciju u crkvi San Clemente u Rimu. Priključuje im i primjerak s portala trogirske katedrale. Literarni predložak radije vidi u pjesmi V. Fortunata (530. - 607.) Lustra sex qui jam peregit.

Drugo pitanje koje pokreće L. Crusvar jest činjenica da u Zadarskoj nadbiskupiji postoje četiri križa (Nin, Božava, Kali, Ugljan) na kojima je Kristov korpus vrlo srodan tršćanskome. Na tu je činjenicu davno upozorio Ivo Petricioli koji je zadarske križeve prvi doveo u vezu s tršćanskim, što je i sam autor ovoga članka svojevremeno usvojio, doduše s oprezom (Jakšić, 2004). L. Crusvar koja usvaja Petriciolove komparacije, posve neopravdano s njima povezuje primjerak iz tršćanske opatije San Cipriano koji međutim pripada jednoj posve drugoj skupini o kojoj je temeljitije pisano u Jakšić 2010. Istovremeno kaže da je riječ o matrici za iskucavanje korištenoj na „cijelom prostoru koji je izložen mletačkom utjecaju“.

Detaljna komparaciji tršćanskog primjerka prema zadarskima pokazuje međutim da križ Alde Zuliani nije iskucan po istoj matrici kao zadarski primjerci. Autor pritom ukazuje na ugovor s zadarskim zlatarom Pavlom Petrovim iz Kotora o izradi križa u Božavi 1407. godine, a tvrdi da je ninski primjerak kojega potpisuje neki Stjepan načinio po istoj matrici Pavlov brat brat Stjepan Petrov iz Kotora koji je međutim umro već prije 1405. godine.

U to doba ni Zadar i Trst nisu u sastavu Mletačke republike niti su pod njezinim utjecajem, pa je stoga posve neprimjerena tvrdnja L. Crusvar o matricama korištenim na „cijelom prostoru koji je izložen mletačkom utjecaju".

Ključne riječi: srednjovjekovno zlatarstvo, srebrna raspela, Zadar, Trst, Alda Zuliani, Stjepan i Pavle iz Kotora

${ }^{44}$ Ivi., p. 26. 